\title{
Experimental and numerical simulation study of perforation effect of steel pipes subject to the impact loadings of ASC and LSC jets
}

\author{
Fuyin $\mathrm{Gao}^{1}$, Chong $\mathrm{Ji}^{2}$, Jianyu $\mathrm{Wu}^{3}$, Xinghua $\mathrm{Li}^{4}$, Yang $\mathrm{Yu}^{5}$ \\ College of Field Engineering, The Army Engineering University of PLA, Nanjing, 210007, China \\ ${ }^{2}$ Corresponding author \\ E-mail: ${ }^{1472564948 @ q q . c o m,{ }^{2} 2468645816 @ q q . c o m,{ }^{3} \text { blastingjar@163.com, }{ }^{4} 379523589 @ q q . c o m,}$ \\ 5962823438@qq.com
}

Received 6 September 2018; accepted 10 October 2018

DOI https://doi.org/10.21595/vp.2018.20205

Check for updates

Copyright (C) 2018 Fuyin Gao, et al. This is an open access article distributed under the Creative Commons Attribution License, which permits unrestricted use, distribution, and reproduction in any medium, provided the original work is properly cited.

\begin{abstract}
The perforation effect of steel pipes subjected to the circular-shaped charge (ASC) and linear-shaped charge (LSC) jet were studied by experimental research, and the explicit nonlinear dynamic finite element computer code LS-DYNA was adapted to study the nonlinear responses of the steel pipes, which subjected to the impact of the two different jets, using Lagrangian-Eulerian coupling method. The deformation process and the stress of the steel pipes were described and analyzed, and the simulation results are in good agreement with the experiment data. The studies indicated that under the impact of ASC jet, the steel pipe got a circular incision and a deformation process of local perforation, flocculent shear lip forming and axial shock. Under the impact of LSC jet, the steel pipe got a ship-type incision and a deformation process of coupling of local perforation and dent, whole bending and radial shock. The formation of flocculent shear lip attributes to the radial stress concentration. Under the impact of LSC jet, the whole bending leads to the axial stretch and tearing of the cut tip, and there is a bigger radial plastic deformation area than the damage effect for the impact of ASC jet.
\end{abstract}

Keywords: shaped charge jet, damage effect, steel pipes, numerical simulation.

\section{Introduction}

Metal pipe or cylindrical shell is widely used in military and civil fields due to its special structure. In the engineering practice, the steel pipe components damaged due to natural and man-make damage can shape cutting way to realize fast repair. In addition, the separation of major steel pipe components, using shaped charge cutting technology, can greatly improve the effect of damage to the target as a special common blasting way. However, different structures and uses of steel pipe components need to cut in different ways, and its damage effect has the corresponding requirements. At present, the research on metal pipe damage effect in the jet impact focused mainly on experimental methods, including Hu Wenjun [1], Jin song [2] using circular shaped cutter for cutting experiments conducted to study the waste nuclear facilities, and Ren Jianxin [3] made ammunition destruction experiment by using linear shaped cutting. While the experiment achieved good damaging effect, but in-depth analysis of dynamic response for the pipe subjected to the jet impact is little considered.

For the damage effect of the pipe structure subjected to explosion impact loading, Ji Chong [4] and others studied dynamic response of the large diameter steel pipe subjected to out contact explosion loading through combination with experimental test and numerical simulation, and analyzed the bulge, spallation and fragmental forming of the pipe. D. Colombo [5] made the numerical simulation research of the different processes of wall-thickness pipe deformations under the projectile impact at different incident angle, observed the phenomenon such as lip and lateral tear. Tomasz [6] made the theoretical and experimental research of the petal shaped perforation under explosion impact load. Shen Xiaojun [7] et al. studied the influence of different fragments shape, Angle, mass and speed on the damage effect; and failure criterion of the thick wall steel 
pipe is given. The above research provides an important reference for the dynamic response of metal pipe (plate) under impact load.

The circumferential and axial cutting experiments of the steel pipe with the same structure and material made in this paper. The deformation and penetration damage effect of the pipe under the two kinds of jet impact by using nonlinear finite element program LS-DYNA in combination with the ALE arbitrary Lagrangian-Eulerian fluid-structure coupling algorithm was studied, the steel pipe deformation characteristics in two kinds of cutting modes were compared and analyzed.

\section{Experiment}

The experimental setting, as shown in Fig. 1, shows that the steel pipe material to cut is Q235 seamless steel, the outer diameter is $40 \mathrm{~cm}$, the wall thickness is $1 \mathrm{~cm}$, and the axial length is $1 \mathrm{~m}$. In the experiment, the radial inner ring and the linear shaped cutter are used, and the material composition and the section size of which are the same. Cutter are placed close to the lateral wall, circular cutter length is the same as the steel pipe girth, linear cutter, $49 \mathrm{~cm}$ long, cutter adopts single point initiation, considering the end initiating may cause "end effect" [8], linear shaped charge cutter initiation point is set in the center of the upper ridge. A wedge cutter section shown in Fig. 2. The shell and the shaped charge liner materials are lead antimony alloy (lead/antimony/other ingredients $=90 / 2 / 8)$. The internal charge line density for pressing 8701 charge is $326 \mathrm{~g} / \mathrm{m}$, the thickness of the shell is $2 \mathrm{~mm}$, the top thickness is $0.8 \mathrm{~mm}$, bus length is $14.1 \mathrm{~mm}$, the outer wall opening Angle $110^{\circ}$, the inner wall of the opening angle is $95^{\circ}$, the fixed charge height is $3.5 \mathrm{~mm}$, and the opening bottom diameter is $22.5 \mathrm{~mm}$.

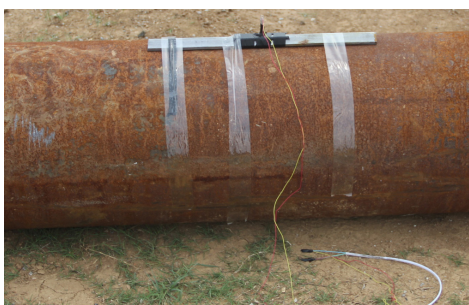

a) Axial cutting

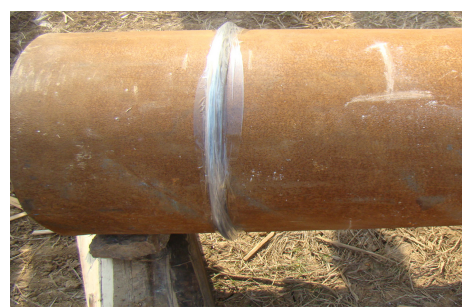

b) Circular cutting

Fig. 1. Experiment setup

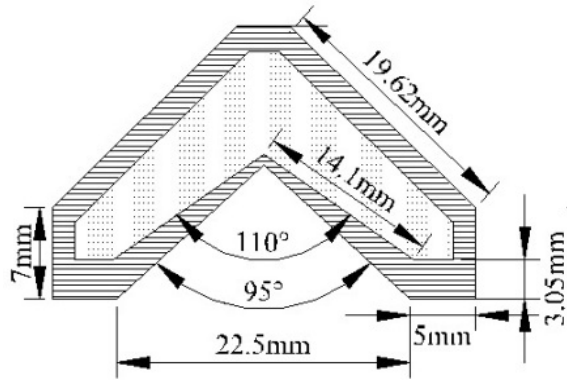

Fig. 2. Schematic diagram of the shaped charge section

\section{Numerical calculation model}

The dynamic process of the pipe subjected to the shaped steel pipe jet impact can be a very good analysis of the physical process, involving in the large pipe body deformation, the flow of the jet and detonation products and high amplitude of shock wave, many substance Eular materials and Largange structure coupled method.

Numerical calculation model shown in Fig. 3, which included air, 8701 of explosives and charge line (shell), air, explosive and charge line modeling used Eular grid, steel pipe modeling 
used Largange grid. The process of the steel pipe impacted by the shaped jet, which used the algorithm of fluid-structure interaction and the hourglass control. Considering the symmetry of the physical model, the prototype 1/2 model used for numerical calculation. In order to save computing resources, mesh optimization carried out for the model. Dense mesh used in the large deformation area of the air and pipeline, and the remaining part of the pipeline is sparse grid. To avoid the pressure to reflect on the boundary, it applied pressure to discharge boundary conditions on the boundary nodes. The calculation model adopts g-cm-s unit system.

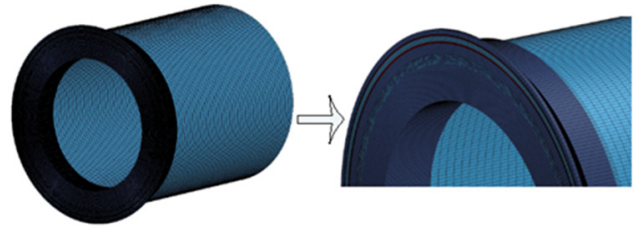

a) Circular cutting model

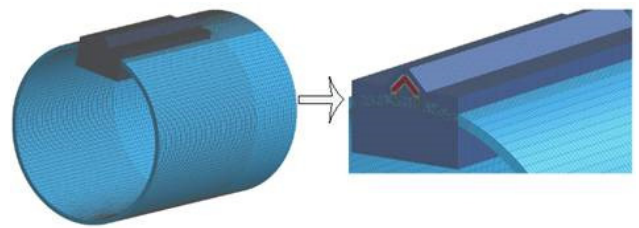

b) Axial cutting model

Fig. 3. The finite element model

\section{Numerical simulation results and analysis.}

\subsection{Circular cutting}

As shown in Fig. 4, under the impact of the circular shaped jet, the steel pipe body was radial fully throughout. The whole fracture into two parts, incision in place near the pipe material is introversion, outer incision is relatively smooth, the inside of which is the relatively rough, flocculent double lip formed, numerical simulation is closer to experimental results. The deformation process of steel pipe under circular cutting shown in Fig. 5. The numerical results show that the deformation of the steel pipe has undergone local penetration, the flocculation of the lip and the axial vibration. Jet around $35 \mu$ s breakdown steel pipe, steel pipe only appeared in the jet impact region occurred severe plastic deformation, formed a circular incision. The incision area materials impacted the detonation products and shaped jet, under high temperature and high pressure, produce thermoplastic instability [10], approximately formed flow body, then outer incision is introversion. Because the steel pipe has a certain length, axial boundary constraints is stronger, so steel pipe axial flip terminated after a certain distance.

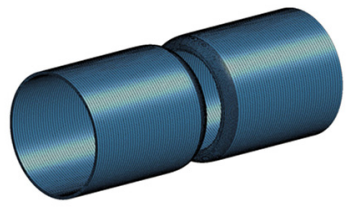

a) Circular incision

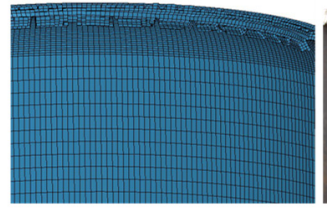

b) Flocculent lip

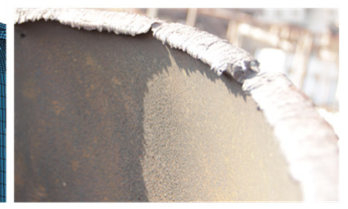

Fig. 4. Damage effect of steel pipe subjected to ASC jet

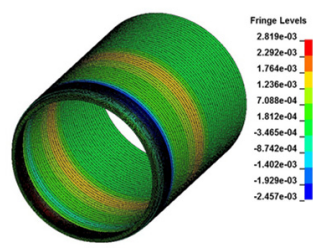

$t=35 \mu \mathrm{s}$

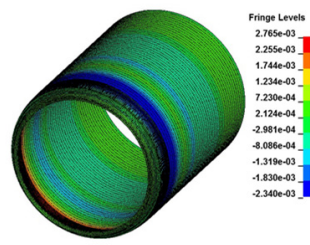

$t=100 \mu \mathrm{s}$

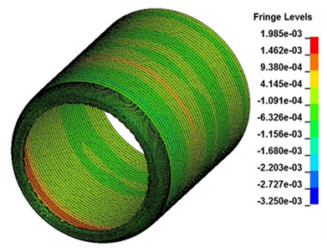

$t=500 \mu \mathrm{s}$

c)

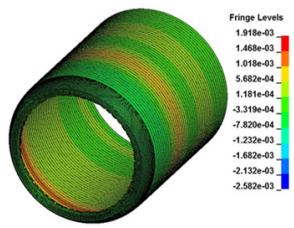

$t=3000 \mu \mathrm{s}$

d)

Fig. 5. Damage process of steel pipe subjected to the impact of ASC jet 
The circumferential cutting is axisymmetric, and can reduce to the two-dimensional problem of the target of the jet perforating steel, without considering the circumferential tensile stress. In the penetrating vicinity of the pipe body, the axial and radial stress curves obtained from a certain distance, at a certain distance along the axial plane, as shown in Fig. 6 and Fig. 7. The stress curve can see that the local plastic deformation stage lasted about $500 \mu \mathrm{s}$. The incision introversion in the process of the deformation is outward, and the material element stress-state is from compression to tension, and tensile stress along the axial direction change into a decreasing trend, and the extension of radial stress first increases and then decreases.

The unit pressure of $0.05 \mathrm{~cm}$ distance from the incision in $50 \mu$ s drop to 0 , shows that at this time the unit has failed, $1.6 \mathrm{~cm}$ distance unit from the incision in $400 \mu \mathrm{s}$, which the axial stress is from tension to compression, radial tensile stress reaches the maximum. After $500 \mu$ s axial force occurred two times jump, the radial force from tension to compression, at this time near the incision units caused by the stress concentration near the pipe material inside the root fracture turned inward to form flocculent double lip. The numerical simulation results show that the process approximately lasts $800 \mu \mathrm{s}$, and then the steel pipe enters the unloading stage. The pipe oscillates and moves a certain distance along the axis under residual stress.
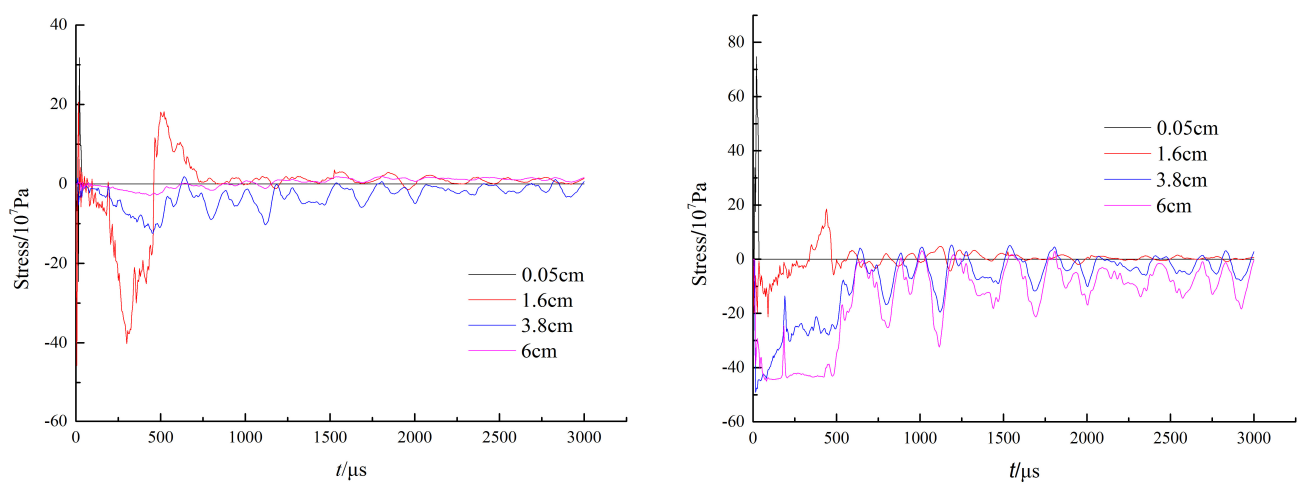

Fig. 6. Radial stress-time curve of steel pipe element Fig. 7. Axial stress-time curve of steel pipe element

\subsection{Axial cutting}

Axial cutting effect shown in Fig. 8. Under the effect of axial impact jet, the steel pipe surface to form a long $52.6 \mathrm{~cm}$, center width $5.6 \mathrm{~cm}$ ship shape incision, steel material extended radial flip, formed double lip section with the width top and narrow bottom. It measured the plastic deformation transverse width is about $10.5 \mathrm{~cm}$ in the maximum plastic area, except for formation of the rough edge due to jet extrusion. The incision inferior is no obvious flocculent double lip compared with the circular cutting. From the surface, the incision made up of a linear body part and two boat tips. From the side, a wide V-shape raised from the middle to both sides.
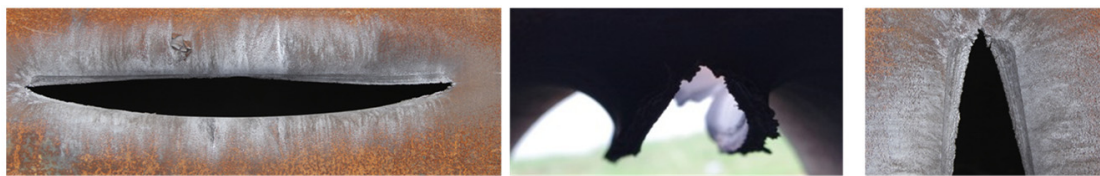

a) Experiment

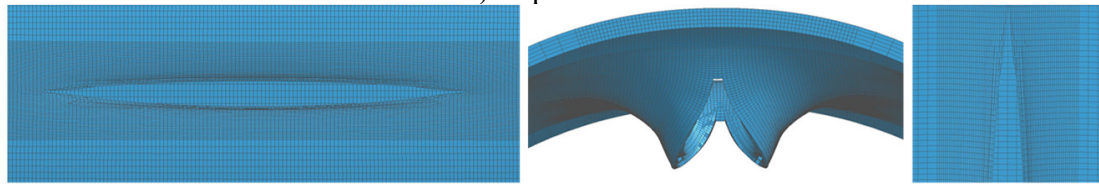

b) Numerical calculation

Fig. 8. Damage effect of steel pipe subjected to the impact of LSC jet 
The deformation process of steel pipe under axial cutting shown in Fig. 9. The deformation of the steel pipe has undergone three stages: the local penetration and the depression coupling, the overall bending plastic deformation and the radial shock. Pipe body is quickly penetrated under the shaped charge jet, and hoop stress is generated because of the arc structure of the steel pipe, as shown in Fig. 10(a). The pipe material unit is from compression to tension changed from the cross section of the lining axis outward, and the incision generated like "air wedge" effect under the circular tensile stress, the detonation products and the wall of residual jet, and the incision was quickly torn a dramatic turn and violently flip along the radial. In compared with the circular cutting, the formation of the radial lip is much more obvious, and the overall plastic deformation area of the cross section is relatively bigger. The numerical simulation results show that local shaping plastic deformation stage lasted roughly $500 \mu \mathrm{s}$ in axil cutting, which is the same of circular cutting.

There is certain axial extension speed of linear shaped charge jet. The Fig. 10(b) shows, under jet impacting the incision appear tensile trend from the blasting center along axis in local through coupling with sag phase, and from the detonation center terminal direction of incision. The variation trend of axial tensile stress is firstly increased and then decreased. It is because in the early stage of the jet forming the charge interior experiences the transformation from the initial internal spherical detonation wave to sliding detonation [11]. Axial extension velocity and the radial jet penetration velocity increased gradually and relatively stable, then end sparse wave propagates along axial inward, sliding detonation wave intensity is reduced, axial and radial jet penetration ability is weakened, led to the incision width is gradually narrow from the center to both ends. The cutting effect of end material is not ideal, and the axial tensile effect of pipe material unit in near the incision end decreased significantly.

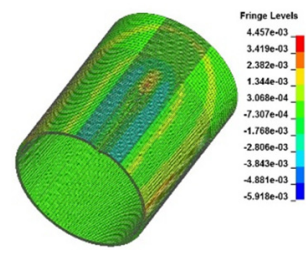

a)

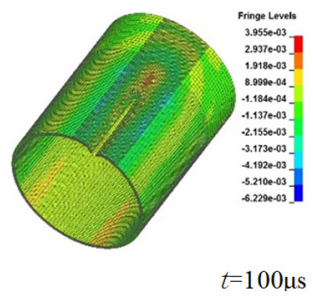

b)

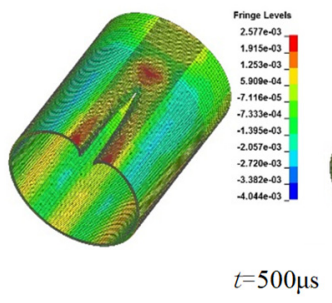

c)

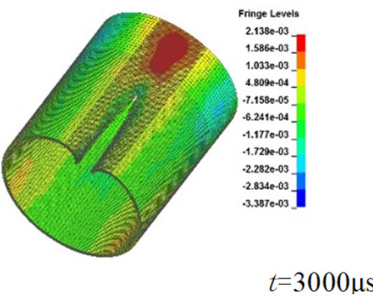

d)

Fig. 9. Damage process of steel pipe subjected to the LSC jet

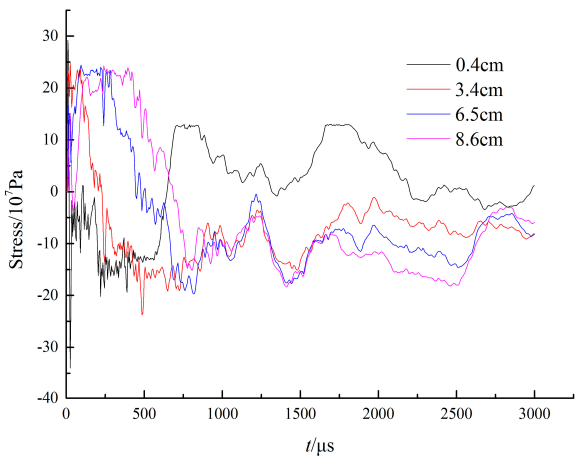

a) Circular

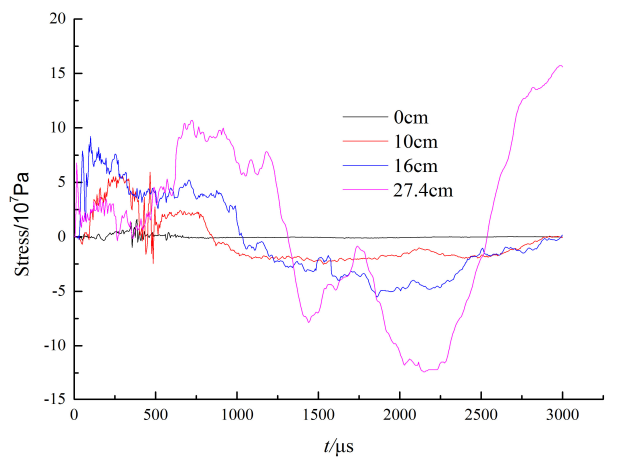

b) Axial

Fig. 10. Stress-time curve of steel pipe element subjected to the impact of LSC jet

After the local plastic deformation, steel pipe still has a larger kinetic energy, and the extension of incision greatly weakened the overall pipe stability. The whole deformation is generated along radial pipe body, when the axial tensile pipe body appear at the same time, which makes the incision end unit $(27.4 \mathrm{~cm})$ to produce stress concentration, incision end tears (Fig. 8). After the 
overall deformation, the pipe enters the unloading process and the radial oscillation occurs under residual stress.

\section{Conclusions}

1) The deformation processes of steel pipe subjected to the circular and linear shaped charge jet impact were investigated. Experimental and numerical results show that the dynamic response of the steel pipe under the jet impact can accurately been simulated through the ALE coupling algorithm, which is a useful supplement in error allowed range that can be used as an experimental method.

2) In the condition that the cutter tightly placed, the steel pipe impacted by the circular shaped charge jet, forms a circular incision. It experienced three stages of deformation, including local plastic deformation, flocculent lip forming and axial oscillation. The formation of flocculent shaped lips caused by radial stress concentration.

3) Under the influence of linear shaped jet impaction, the ship shaped incision of steel pipe has experienced three stages of deformation, including local penetration and sunken coupling, the whole bending plastic deformation and radial oscillation. The whole deformation causes the pipe to extend the axial tension and tear effect at the notch tip. The results provide a useful reference for understanding the dynamic effect of steel pipe subjected to the impact of shaped charge jet.

\section{Acknowledgements}

This research was financially supported by the National Nature Science Foundation of China, Nos. 11102233,51678567 and 51608530.

\section{References}

[1] Wu Wen-jun, Zhang Yu-ren Experimental study of nuclear facilities pipeline under blasting cutting. Blasting, Vol. 2, 1994, p. 75-77.

[2] Kong Jin-song, Liu Shao-tian, Zhang Sheng-jiang, et al. Development of ringed-blast-cutter and its application in Decommission of nuclear installation. Nuclear Power Engineering, Vol. 3, Issue 22, 2001, p. 276-288.

[3] Ren Xin-jian, Li Lin, Wang Jian-hui Design of cumulative jet to eliminate unexploded bomb. Blasting, Vol. 4, Issue 25, 2008, p. 82-84.

[4] Ji Chong, Long Yuan, Fang Xiang, et al. Numerical simulation of large-aperture steel pipe subjected to contact explosion loading. Journal of Vibration and Shock, Vol. 16, Issue 31, 2012, p. 72-76.

[5] Colombo D., Giglio M. Numerical analysis of thin-walled shaft perforation by projectile. Computers and Structures, Vol. 85, 2007, p. 1264-1280.

[6] Wierzbicki Tomasz Petalling of plates under explosive and impact loading. International Journal of Impact Engineering, Vol. 22, 1999, p. 935-954.

[7] Shen Xiao-jun, Zhang Peng-xiang, Sun Tao, et al. Research on thick barrel's damages due to impaction by fragment of high explosive projectile. Acta Armamentarium, Vol. 26, Issue 4, 2005, p. 438-442.

[8] Wu Shuang-zhang, Gu Wen-bin Experimental and three-dimensional numerical simulation for influence of end constraint jet formation of cuneiform cover cutter. Chinese Journal of Energetic Materials, 2013.

[9] Kohn B. J. Compilation of Hugoniot Equations of State. Air Force weapons Laboratory Report AFWL-TR-69-38, 1969.

[10] Hoggatit C. R. Fracture behavior of tubular bombs. Journal of Applied Physics, Vol. 39, 1968, p. $1856-1862$.

[11] Chu Chao-hsiang, Jiang Da-he On the motion of flying plate under explosive attack. Applied Mathematics and Mechanics, 1980, p. 293-304. 